\title{
Characterisation and use of $\beta$-lactoglobulin fibrils for microencapsulation of lipophilic ingredients and oxidative stability thereof
}

\begin{abstract}
There is a growing interest in using fibrils from food grade protein, e.g. $\beta$-lactoglobulin, as functional ingredients. In the present study, the functionality of fibrillar $\beta$-lactoglobulin from whey protein isolate (WPI) was compared to native WPI in terms of interfacial dilatational rheology and emulsifying activity at acidic conditions ( $\mathrm{pH} 2.0$ and 3.0). We report here for the first time data on microencapsulation of fish oil by spray-drying as well as oxidative stability of the oil in emulsions and microcapsules in dependence of WPI conformation. WPI fibrils exerted a significantly higher elasticity at the oil-water $(\mathrm{o} / \mathrm{w})$ interface and a better emulsifying activity at a fixed oil content compared to native WPI. Microencapsulation efficiency was also higher with fibrillar WPI (>95\%) compared to native WPI ( 90\%) at $\mathrm{pH}$ 2.0 and a total oil and protein content of $40 \%$ and $2.2 \%$, respectively, in the final powder. The oxidative deterioration was lower in emulsions and microcapsules prepared with fibrillar than with native WPI. This was attributed to improved interfacial barrier properties provided by fibrils and antioxidative effects of coexisting unconverted monomers, particularly hydrophilic peptides.
\end{abstract}

Keyword: Fibrils; $\beta$-Lactoglobulin; Homogenisation; Microencapsulation; Spray drying 